\title{
Dynamic Response in Transient Operation of a Variable Geometry Turbine Stage: Influence of the Aerodynamic Performance
}

\author{
Nicolas Binder, Jaime Garcia Benitez, and Xavier Carbonneau \\ Département d'Aérodynamique, Energétique et Propulsion, Université de Toulouse, ISAE, BP 54032, \\ 31055 Toulouse Cedex 4, France
}

Correspondence should be addressed to Nicolas Binder; nicolas.binder@isae.fr

Received 8 April 2013; Accepted 4 July 2013

Academic Editor: Dimitrios T. Hountalas

Copyright (C) 2013 Nicolas Binder et al. This is an open access article distributed under the Creative Commons Attribution License, which permits unrestricted use, distribution, and reproduction in any medium, provided the original work is properly cited.

The transient response of a radial turbine stage with a variable geometry system is evaluated. Mainly, the consequences of the variations of the aerodynamic performance of the stage on the response time are checked. A simple quasi-steady model is derived in order to formalize the expected dependences. Then an experimental campaign is conducted: a brutal step in the feeding conditions of the stage is imposed, and the response time in terms of rotational speed is measured. This has been reproduced on different declinations of the same stage, through the variation of the stator geometry, and correlated to the steady-state performance of the initial and final operating points of the transient phase. The matching between theoretical expectation and results is surprisingly good for some configurations, less for others. The most important factor identified is the mass-flow level during the transient phase. It increases the reactivity, even far above the theoretical expectation for some configurations. For those cases, it is demonstrated that the quasi-steady approach may not be sufficient to explain how the transient response is set.

\section{Introduction}

Many applications make use of the interesting properties of radial inflow turbines, namely, the compactness and the ability of reaching high pressure ratios for acceptable efficiencies. The description of the radial turbines operating in steady flows has been widely presented in the literature $[1,2]$, together with an extensive description of the flow pattern, even for off-design conditions (e.g., in [3]). Some design processes have also been presented to ensure the good performance of the energy-recovering process of the stage (see $[4,5])$. The favourable pressure gradient due to the expansion of the flow through the turbine stage stabilizes the boundary layer behaviour and makes separations unlikely if the guidance of the flow is appropriate. As a consequence, a one-dimensional description of the flow is adequate to reach an accurate description of the radial turbine functioning [4]. This is transposable to operational and geometric off-design operation [6]. But the problem gets more complex when the inlet conditions are time dependent. Most of the contributions analysing radial turbines operating in unsteady flows are found in the literature dedicated to possible turbocharging applications. The turbine stage experiences some severe pulsating inlet conditions due to its position in the exhaust manifold. The research activity in that field is currently very active. A review of the state-of-the-art and of the main contributors of the field can be found in $[7,8]$. The fact that the rotor part of the stage operates in a quasi-steady manner is one of the most significant conclusions of the different studies. Even if this assessment needs to be generalized (far from the design point, the rotor is likely to be more sensitive to some unsteady processes), it points out that the performances of the static part of the stage (volute and stator) are strongly affected by the time-variation of the inlet conditions.

Another kind of time-dependent operation is defined when dealing with transient phases of the turbine stage. 
There again, most of the research is motivated by automotive propulsion, since the turbocharger often experiences such a kind of request on a typical run. The literature depicts how the response time of the turbocharger impacts the transient response of the engine. This is presented through the modelling of the engine dynamic response when coupled to a turbocharger, compared with experimentations (in $[9,10])$. The turbocharger is then modelled as a system having a first-order transfer function, which is a good representation according to [11]. The general assessment is that the mass-moment of inertia of the rotating assembly is the most dominant factor influencing the response time of the turbocharger (e.g., in [12]). A direct consequence of this variation of the massmoment of inertia of the turbocharger shaft on the engine overall response is presented in [10]. The issue of reducing the mass-moment of inertia is that the turbine stage must sustain high temperatures and thus requires high-density materials of construction. Some solutions, such as scalloping, are recommended to decrease as much as possible this moment of inertia [12], even if it deteriorates the aerodynamic efficiency. Anyway, the presumed importance of the moment of inertia of the turbocharger shaft is still in discussion. A great part of this discussion is focused on the overall reactivity of the engine, for which the mechanical, chemical, and thermodynamic inertia of the engine itself should be considered (according to $[9,13,14]$ ). A marginal part of the discussion deals with the isolated turbocharger to understand how its own transient response is affected by other factors. One solution to increase the reactivity is the use of variable geometry devices either on the compressor or on the turbine stage. This is demonstrated in different studies $[13,15,16]$ and highlights once again the importance of the static part of the stage, here on the dynamic response of the rotor. The control strategy of the variable geometry device is then crucial as demonstrated by [13]. In [14] is mentioned the fact that the efficiency of the stage is of the same order of importance as the mass-moment of inertia in the establishment of the response time. It is indeed surprising that such an extensive knowledge of flow pattern in radial turbines, as described above, is that poorly correlated to the dynamic response in transient operation. No clear correlation between the dynamic performance and the efficiency of the turbine is presented. Since the definition of efficiency in unsteady flows is a complex matter [17], the only indicator available is the efficiency measured in steady flows. The comparison of time scales of the stage (rotor rotation, propagation of the pressure, or mass wave in the stator) to that of a typical transient request tends to validate this "steady" indicator as representative of the performance. But this is questionable since a pressure wave will not be instantaneously distributed all around the rotor and will definitely affect the boundary layers in the stage. The present work is a first contribution to understand how the efficiency of a radial turbine stage interferes with its dynamic response for different geometric configurations. The first part of the paper focuses on the theoretical background in order to identify the most relevant factors of the problem. The second part of the paper presents the experimental approach and the facility. The last part of the paper presents the results which are discussed in the conclusion.

\section{Theoretical Background}

The time evolution of the rotational speed during transient operation is based on a rather simple mechanical equilibrium. If the turbine stage is connected to another device through a solid crank arm (compressor, electric generator, etc.) for which the torque is quoted $\tau_{B}$, it can be written as follows:

$$
J \cdot \frac{d \omega(t)}{d t}=\tau_{T}-\tau_{B}
$$

Since

$$
\dot{W}=\tau_{T} \cdot \omega,
$$

if there is no gearbox plugged between the two systems, in steady operation both the torque and the rotational speed of the turbine and of the braking device are equal (the contribution of the bearings is taken into account as part of the "braking" device). For the turbine, in steady-state operation, the power extracted from the flow is given by the first law of thermodynamics:

$$
\dot{W}=\eta_{T} \dot{m} C_{p T} T_{t 1}\left[1-\left(\frac{1}{\pi_{T}}\right)^{(\gamma-1) / \gamma}\right] .
$$

This statement forbids the direct integration of (1), because the dependence of the quantities (mass flow, pressure ratio, and efficiency) to the rotational speed is not explicit. In steady-state operation, a complete map is required to identify this dependence; it does not have a simple analytical expression. And in transient operation, the validity of this map is not demonstrated. Equally, the braking work $\left(\dot{W}_{B}\right)$ depends on the rotational speed and also on the characteristics of the device to which the turbine stage is connected.

The exact modelling of the transient behaviour is not the purpose of this work. But a theoretical reference is needed to meet the two main objectives listed here:

(1) the identification of the most influential factors controlling the setting of the response time (in addition to the obvious contribution of the mass-moment of inertia);

(2) the verification of the validity of a quasi-steady approach in transient processes.

In that purpose, the most simple quasi-steady theoretical expression of the response time for transient operation is built. The transient phase is defined as follows: from an initial steady-state operating point (denoted with " $i$ ” indices), a sudden rise (characteristic time of the step $<10 \mathrm{~ms}$ ) of pressure and mass-flow at inlet of the stage leads to a second steady-state operating point (denoted by " $f$ " indices). One has to drastically simplify the problem to integrate (1). Those simplifications are as follows:

(i) an intermediate point is defined (denoted by “ $i$ " indices). The transient phase is then decomposed in two subphases $\left[i-i^{\prime}\right]$ and $\left[i^{\prime}-f\right]$, as illustrated in Figure 1; 


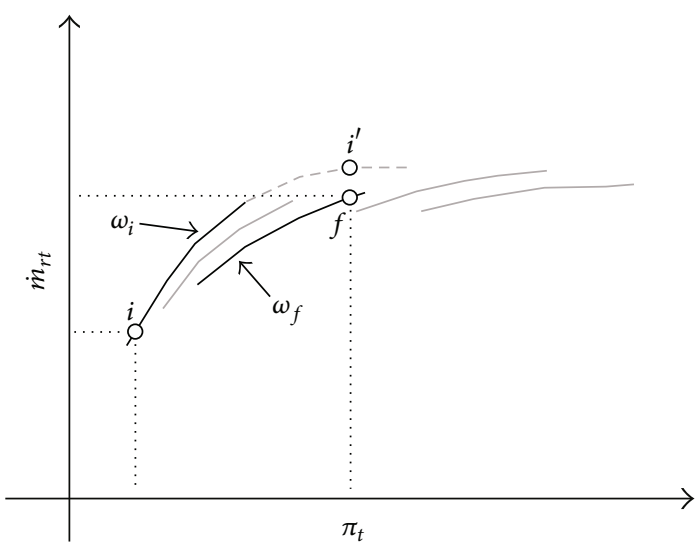

(a) $i-i^{\prime}-f$ phases in the turbine map

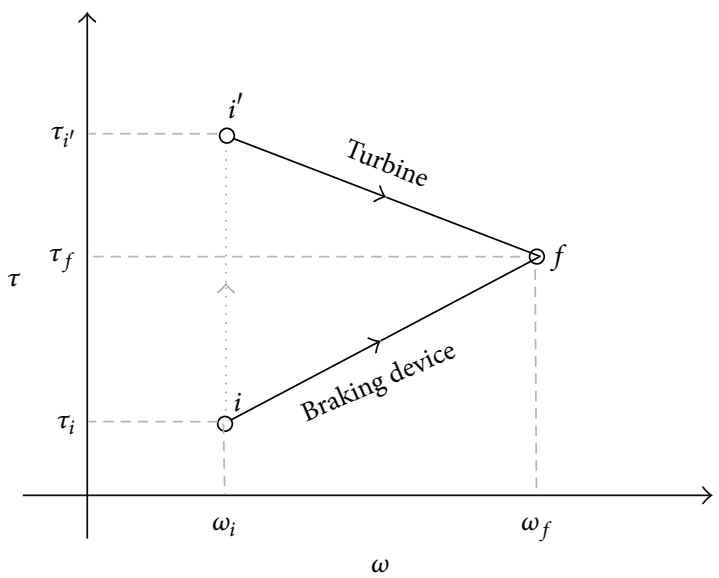

(b) $i-i^{\prime}-f$ torque-to-rotational speed characteristics

FIGURE 1: Decomposition of the transient phase from initial $(i)$ to final $(f)$ operating point through intermediate $\left(i^{\prime}\right)$ operating point.

(ii) the first subphase $\left(\left[i-i^{\prime}\right]\right)$ considers the instantaneous variation of the inlet pressure and the mass flow to their final values, at a fixed rotational speed. This is supported by the characteristic velocity of propagation of the pressure perturbation inside the turbine stage which is near the speed of sound (as presented in [7] and discussed in [8]); the time scale is thus shorter than the one of the shaft velocity variation, which keeps its initial value. Then $\omega_{i^{\prime}}=\omega_{i}$, $\dot{m}_{i^{\prime}} \approx \dot{m}_{f}$, and $\pi_{T, i^{\prime}} \approx \pi_{T, f}$. The torque $\tau_{i^{\prime}}$ is discussed later;

(iii) the second subphase $\left(\left[i^{\prime}-f\right]\right)$ consists in the variation of the rotational-speed due to the difference of torque between turbine and braking device;

(iv) a linear evolution of the torque-to-rotational-speed characteristic for both the turbine and the braking device is assumed since it is difficult to evaluate it $a$ priori (see Figure 1).

In that quasi-steady situation, a simple expression for both the turbine and braking torques can be obtained. On the braking device side, the functioning characteristic is linear and must be compatible with the final operating point of the turbine (to reach the power equilibrium in steady state operation). It means that

$$
\tau_{B}(t)=\frac{\tau_{T_{f}}}{\omega_{f}} \omega(t) .
$$

The same treatment is applied on the turbine side, namely, linear variation and compatibility with the final operating point:

$$
\tau_{T}(t)=\tau_{T, f}+\frac{\tau_{T, f}-\tau_{T, i^{\prime}}}{\omega_{f}-\omega_{i}}\left(\omega(t)-\omega_{i}\right)
$$

Equation (1) then gives

$$
\begin{array}{r}
J \frac{d \omega(t)}{d t}+\left(\frac{\tau_{T, f}}{\omega_{f}}-\frac{\tau_{T, f}-\tau_{T, i^{\prime}}}{\omega_{f}-\omega_{i}}\right) \omega(t) \\
=\frac{\omega_{i}}{\omega_{f}-\omega_{i}}\left(\tau_{T, i^{\prime}}-\tau_{T, f}\right)+\tau_{T, i^{\prime}} ;
\end{array}
$$

this equation reduces to a first-order differential equation as follows:

$$
\frac{d \omega(t)}{d t}+\left[\frac{\omega_{f} \tau_{T, i^{\prime}}-\omega_{i} \tau_{T, f}}{J \omega_{f}\left(\omega_{f}-\omega_{i}\right)}\right] \omega(t)=K,
$$

where $K$ is a constant. For such an equation it is possible to identify a time parameter $\kappa$ which is representative of the response time of the shaft. Its expression is

$$
\kappa=J \frac{\omega_{f}\left(\omega_{f}-\omega_{i}\right)}{\omega_{f} \tau_{T, i^{\prime}}-\omega_{i} \tau_{T, f}} .
$$

An estimation of the torque $\tau_{T, i^{\prime}}$ generated by the turbine at point $i^{\prime}$ is difficult to give. Such a transient operating point is hardly accessible by measurement. The corresponding steady-state operating point, if it exists, is generally out of the range of conventional test benches, since a large overlapping zone between the speed-lines of the map is required to capture it. This is hardly possible when the braking device is a compressor, limited by blockage and surge/stall regions. A last estimation is then proposed for the torque at this intermediate step of the transient, in order to close the model as simply as possible.

(v) The variations of mass-flow, efficiency, and pressure ratio are supposed to be small between the points $i^{\prime}$ and $f$. Then $\dot{W}_{i^{\prime}} \approx \dot{W}_{f}$, which leads to the following approximation:

$$
\tau_{i^{\prime}} \approx \frac{\omega_{f}}{\omega_{i}} \tau_{f} .
$$


The time parameter is written as follows:

$$
\kappa=J \frac{\omega_{f}}{\tau_{T, f}} \sigma,
$$

where $\sigma$ is a parameter only depending on the initial and final rotational speeds. It is written as follows:

$$
\sigma=\left(\frac{\omega_{f}-\omega_{i}}{\omega_{f}^{2} / \omega_{i}-\omega_{i}}\right)
$$

Finally, according to (2) and (3), and to the definition of isentropic speed, one gets

$$
\kappa=\frac{J}{\dot{m}_{f} \eta_{f}} \frac{8}{D_{4}^{2}}\left(\frac{U_{4}}{C_{s}}\right)_{f}^{2} \sigma .
$$

The response time to a brutal modification of the operating point from the steady conditions " $i$ " to the steady conditions " $f$ " obviously depends on moment of inertia of the shaft. But it also depends on the initial rotational speed (through $\sigma$ ) and on the final operating point performance. It means that a given jump of rotational speed will not have the same duration, depending on the region of the map considered (illustrated in Figure 2). According to the simplified model proposed in (12), decreasing the moment of inertia will improve the reactivity of the stage at every position on the map, but a general improvement in the stage efficiency will have the same effect. Otherwise, if a general improvement cannot be achieved, this simple relation points out the fact that the low mass-flow configuration should be favoured in efficiency, since the high mass-flow regions are naturally reactive.

This result has been derived with the restrictive hypotheses above mentioned. The validity of these hypotheses is also dependent on the position of the operating in the map, and on the geometric configuration of the stator. For example, considering the mass flow constant during the subphase $\left[i^{\prime}-\right.$ $f]$ in a transient which is near the blockage region seems more valid than in other regions of the map, or the modification of the stator geometry may shift the reduced tip speed from its optimum value of 0.7 . The real dependence of the transient response on the factors identified still needs to be checked.

\section{Experimental Approach}

The approach is based on a single geometric configuration of the rotating assembly: radial inflow turbine and centrifugal compressor (see Figure 3(a)). The stator of the turbine stage is modified to create different flow configurations and check the dependence of the factors identified in (12). Three modifications are imposed and presented in Figure 3(b): (i) opening angle of the stator blades, (ii) height of the stator blades, and (iii) presence of the spacers and clearances in the stator. The first two modifications aim at the alteration of the mass-flow level inside the stage without changing the final pressure ratio. The last modification interferes with the efficiency of the stage, for the presence of the spacers creates some clearances at the tip and at the hub of the stator

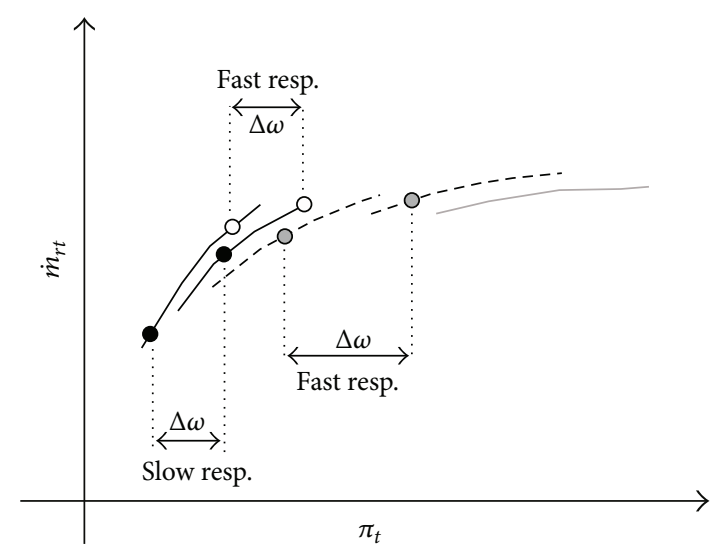

FIGURE 2: Transient response of the stage depending on the position of " $i$ " and " $f$ " in the map for the same rotational speed jump $\Delta \omega$.

blades and generates some losses. It is an artificial method to change the efficiency of a given operating point. When dealing with variable geometry stators, the importance of the reduced section $\left(S_{3}^{*}\right)$ is demonstrated in [6]. This parameter is a nondimensional expression of the throat section of the stator; its expression is given in (13). The setting of this reduced section defines the operating point of the stage. The stator opening angle and height combinations are chosen so that some different geometric configurations generate similar values of reduced section. For most of the configurations, some tests with and without spacer/clearances are conducted. The different geometric configurations are labelled according to the opening position of the stator (1: closed; 2 : intermediate closed/reference; 3: reference...5: opened) and subscripted with the height of the stator (s: small; r: reference; h: high). The configurations tested and the associated values of the parameters are presented in Table 1 :

$$
S_{3}^{*}=\frac{S_{3}}{D_{4}^{2}\left(D_{3} / D_{4}\right) \sin \left(\alpha_{3}\right)} .
$$

3.1. Steady-State Measurements. The "steady-state" identification of the initial and final operating points is obtained on our PTM (Petite TurboMachine) test rig dedicated to turbocharger applications. This steady flow test rig allows either global or local instrumentation of small stages, for a wide range of inlet temperatures (from $20^{\circ} \mathrm{C}$ to $620^{\circ} \mathrm{C}$ ), up to $250 \mathrm{~g} / \mathrm{s}$. The air is supplied by a pressure source $(6$ bars) stabilized by two regulation stages. The compressor and turbine flows are independent. They are thermally isolated from the ambient air and from each other. A specific circuit supplies hot and pressurized oil to the bearings, with a proper regulation. Inlet/outlet pressure and temperature are measured in order to get the performance of both stages. Massflow measurement is operated through a Coriolis flowmeter. Rotational speed is measured using a proximity sensor on the compressor stage. For more details about the test facility, see [18]. The repeatability quality of the experimental device is the good indicator for the experimental uncertainties since the 


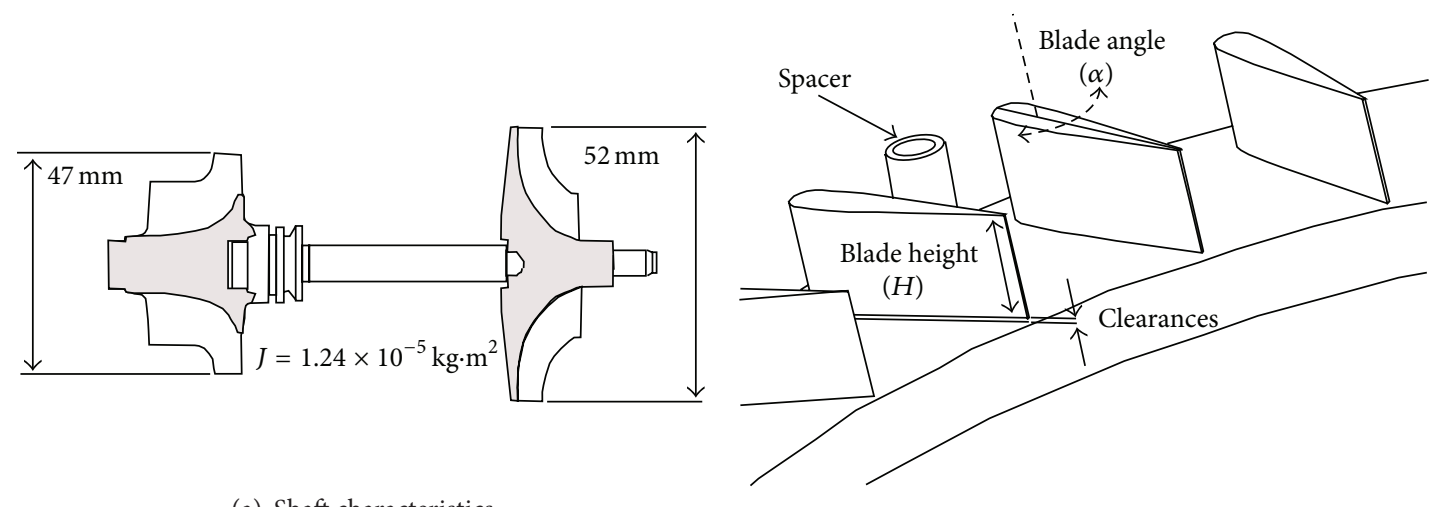

(a) Shaft characteristics

(b) Modifiable parameters of the turbine stage

FIGURE 3: Illustration of the geometric characteristics.

TABLE 1: Characteristics of the stators.

\begin{tabular}{lccc}
\hline Stator & $\Delta \alpha$ & $H / H_{\text {ref }}$ & $S_{3}^{*}$ \\
\hline $\mathrm{lr}$ & $-15^{\circ}$ & 1 & 0.074 \\
$3 \mathrm{r}$ & $0^{\circ}$ & 1 & 0.215 \\
$5 \mathrm{r}$ & $+22^{\circ}$ & 1 & 0.520 \\
\hline $1 \mathrm{~s}$ & $-15^{\circ}$ & 0.71 & 0.047 \\
$2 \mathrm{~s}$ & $-10^{\circ}$ & 0.71 & 0.086 \\
$4 \mathrm{~s}$ & $+10^{\circ}$ & 0.71 & 0.230 \\
\hline $\mathrm{h}$ & $-15^{\circ}$ & 1.13 & 0.086 \\
$3 \mathrm{~h}$ & $0^{\circ}$ & 1.13 & 0.257 \\
\hline
\end{tabular}

experimental study deals with comparative results. The confidence ranges are given in Table 2 for the nondimensional quantities presented in the results.

For machining convenience, the prototype stators were built with aluminum alloy (AU4G) which does not sustain high temperatures. The air-flow temperature supplied to the turbine stage is thus left at ambient value. The different pressure ratio lines are then described $\left(\pi_{t s}\right.$ from 1.35 to 1.6) by modifying the loading of the compressor, which is controlled by a discharge valve.

3.2. Unsteady Measurements. An improvement of the PTM test rig is developed, to meet unsteady measurement requirements. A rapid solenoid valve generates a sharp pressure variation at the inlet of the turbine stage. A flow derivation supplies air for the stage when the valve is closed, so that the rotor never stops. This flow supply defines the initial operating point. Then the valve is opened. A rapid modification of the inlet conditions (the typical time of variation of the inlet pressure is $10 \mathrm{~ms}$ ) forces the turbine stage to reach the final operating point. A typical transient phase imposed to the stage by the valve opening, in terms of initial and final pressure ratios, is from $\pi_{t, i}=1.15$ to $\pi_{t, f}=1.60$. Some unsteady measurement devices were implemented: two fastresponse pressure probes at the inlet of the stage and at the outlet of the compressor stage; a fast-response proximity
TABLE 2: Repeatability uncertainty of the bench.

\begin{tabular}{lcc}
\hline Quantity & $95 \%$ confidence range & \% of ref. conditions \\
\hline$\dot{m}_{r t}$ & $\pm 0.5 \mathrm{~g} / \mathrm{s}$ & $0.5 \%$ \\
$\omega$ & $\pm 50 \mathrm{rad} / \mathrm{s}$ & $0.5 \%$ \\
$\pi_{t}$ & \pm 0.003 & $0.2 \%$ \\
$\eta$ & $\pm 0.5 \mathrm{pts}$ & $0.8 \%$ \\
\hline
\end{tabular}

sensor near the compressor impeller to detect the blade passage.

The pressure sensors are conditioned by in-house amplifiers designed to set the cut-off frequency 10 times greater than the maximum frequency expected in the pressure excitation signal. The sampling frequency of the data acquisition system is set at $200 \mathrm{KHz}$ to ensure an accurate resolution in the observation of the blade passage by the proximity sensor. The raw signal of the blade passage recorded is postprocessed to extract the temporal evolution of the rotational speed.

The initial and final operating points are identified by the use of the steady-state metrology. The extraction of the response time of the shaft is performed through the postprocess of the unsteady measurements. For safety reasons, some regulation of the pressure upstream of the solenoid valve has been implemented. This regulation process is less rapid than the solenoid valve and suffers some lag. This makes the pressure step at the inlet of the stage unperfect (see Figure 4). Thus the response time is not accessible through direct measurements of the raw signal. The shaft should behave like a first-order system according to (7) since the initial and final operating points are fixed. It has thus been decided to use an identification function to express the impulse response of our system. The entry of the system is the pressure rise at inlet, and the response is the rotational speed evolution. The mean square method is applied to match the coefficients to the experimental evolution of the two quantities. The production of the final model is then compared with the data. The matching between the prediction and the experimental data is in the range of $98 \%$ for all of the configurations. The characteristic response time of the first-order model is taken as the estimation of the actual response time of the 


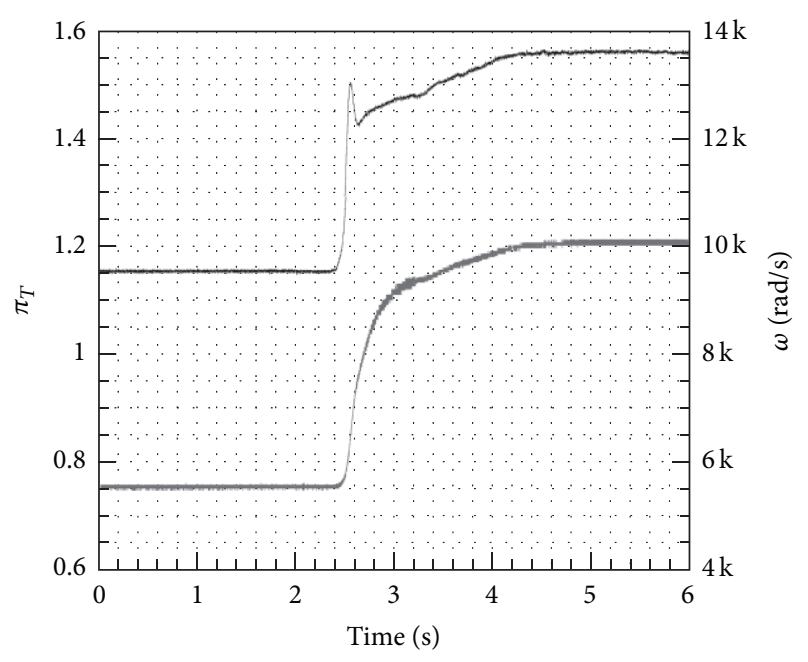

FIgURE 4: Time evolution of the pressure ratio in the stage (upper curve) and of the rotational speed (lower curve).

stage. Ten repetitions of each experiment give access to the standard deviation of the response time and to the associated confidence range at $95 \%$. This range is expressed in the following results as error bars around the response time data (typical value: $\pm 2.5 \mathrm{~ms}$ ).

3.3. Numerical Simulations. The intermediate torque $\left(\tau_{i^{\prime}}\right)$ is only estimated in the model and never measured. As stated above, the validity of the estimation depends on the location of the transient phase in the map and cannot be experimentally confirmed. Some numerical simulations were punctually conducted in order to reach estimations of the torque for some operating points out of reach of the test bench. Those simulations were performed with the Euranus solver, of the Fine/Turbo software suite of Numeca Int. The stator-rotor configuration is meshed with Autogrid 5 (1.5 million cells, $Y^{+}<5$ ). The steady simulations (mixing plane condition at the rotor/stator interface) use the Spalart-Allmaras turbulence model. The inlet total conditions, outlet static pressure, and rotational speed are imposed as boundary conditions.

\section{Results}

In this section, the analysis of the results is divided in two parts. First, one focusses on the factors highlighted by the theoretical development for their supposed importance in the establishment of the response time (mainly the final massflow and the final efficiency). Second, the discrepancy of the theoretical model is analysed to feed the discussion about the relevance of the quasi-static approach for transient operation.

4.1. Identification of the Influential Factors. Figure 5 presents the general influence of both the mass-flow and the efficiencies of the final operating point on the transient response of the stage. The mass-flow and efficiency plotted values are relative to that of the final point for the reference configuration ( $3 r$, with spacers). The steady-state part of the results (Figure 5(a)) shows the performance of the stage for three opening configurations (closed: $1 r$, nominal: $3 r$, and fully opened: $5 r$ ). The value of the final pressure ratio is the same for the three configurations $\left(\pi_{t, f}=1.60\right)$. The results confirm that it is possible to downgrade the efficiency by the use of the spacers and the clearances, without drastic modifications of the operating point. The penalty induced by the spacers and the clearances is far more important in the closed configuration than in the fully opened one. This has already been explained long ago by [19] since the relative importance of the clearances increases as the throat section of the nozzle decreases. In response to the alteration of both the mass-flow and the efficiency levels, the reactivity of the stage is modified as presented in Figure 5(b). The general trend is in agreement with the theoretical expectation: when the mass-flow level in the stage increases at the end of the transient phase, the response time decreases. It also decreases when the efficiency is increased (for the same mass-flow level across the stage), even if the tendency is less clear. For the closed configurations, the theoretical prediction based on the steady-state measurements of the initial and final operating points is surprisingly accurate. This accuracy is lost for the two other configurations, for which the prediction strongly overestimates the response time: the shaft is almost twice more reactive than expected by the model. The strong dependence of the response time on the final operating point definition is examined in Figure 6. The steady-state results show the position of the initial point and that of the three final operating points in the turbine map, for the stage configuration $3 h$. The gap in rotational speed between initial and final operating points is successively increased (from $\Delta \omega$ $=3000 \mathrm{rad} \cdot \mathrm{s}^{-1}$ for transient 1 to $\Delta \omega=6000 \mathrm{rad} \cdot \mathrm{s}^{-1}$ for transient 3 ), together with the final mass-flow rate and final pressure ratio, as shown in Figure 6(a). Figure 6(b) presents the response time of the three imposed transient phases. It decreases despite the apparent increase of the distance in the map between the initial and final points. The model also predicts this trend. Equation (12) shows how the "geographic" distance between the initial and final operating points in the map is irrelevant in the prediction of the response time; the proximity obviously does not ensure a short transient phase. The good reactivity is better ensured by a large mass-flow through the stage at the end of the transient and to a lesser extent by a good efficiency.

The results examined in Figure 6 also reinforce the conclusion that in certain conditions the experimental value of the response time is strongly overestimated by the quasistatic approach. The accuracy of the theoretical prediction is thus investigated.

4.2. Validity of the Quasi-Static Approach. A general evaluation of the theoretical model is proposed in Figure 7. All the configurations are classified in terms of reduced section, as presented in Figure 7(a), where the corrected mass-flow of the final operating point is plotted. This diagram shows the importance of the value of the reduced section $\left(S_{3}^{*}\right)$ for variable geometry stages: for a given pressure ratio, the mass-flow values of the operating point collapse on a single trend. This occurs despite the fact that the different stators 


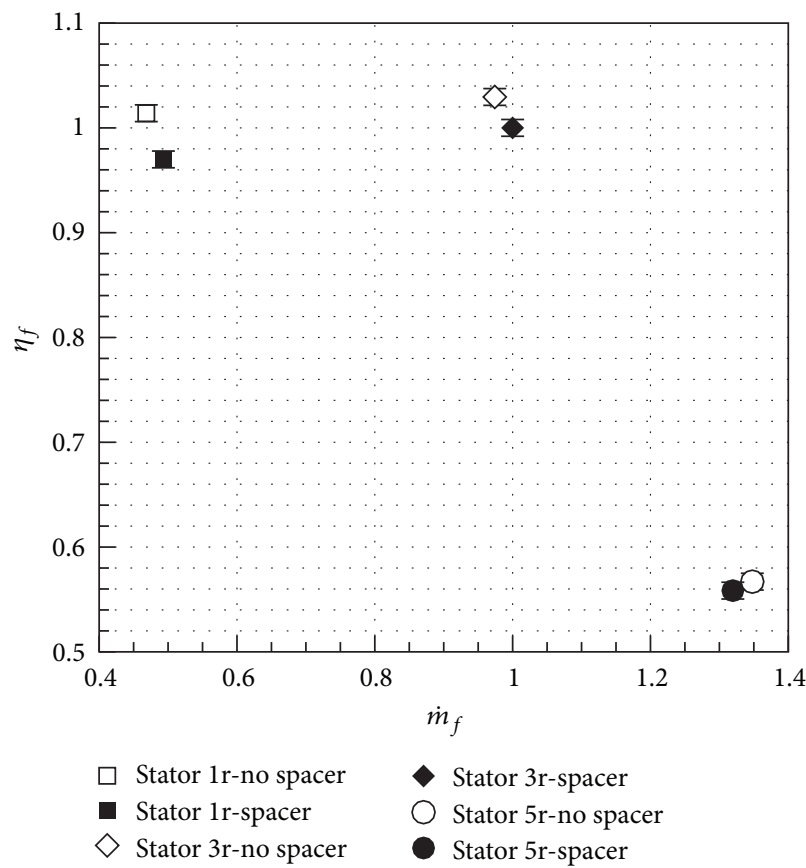

(a) Efficiency as a function of the mass-flow for the final operating point

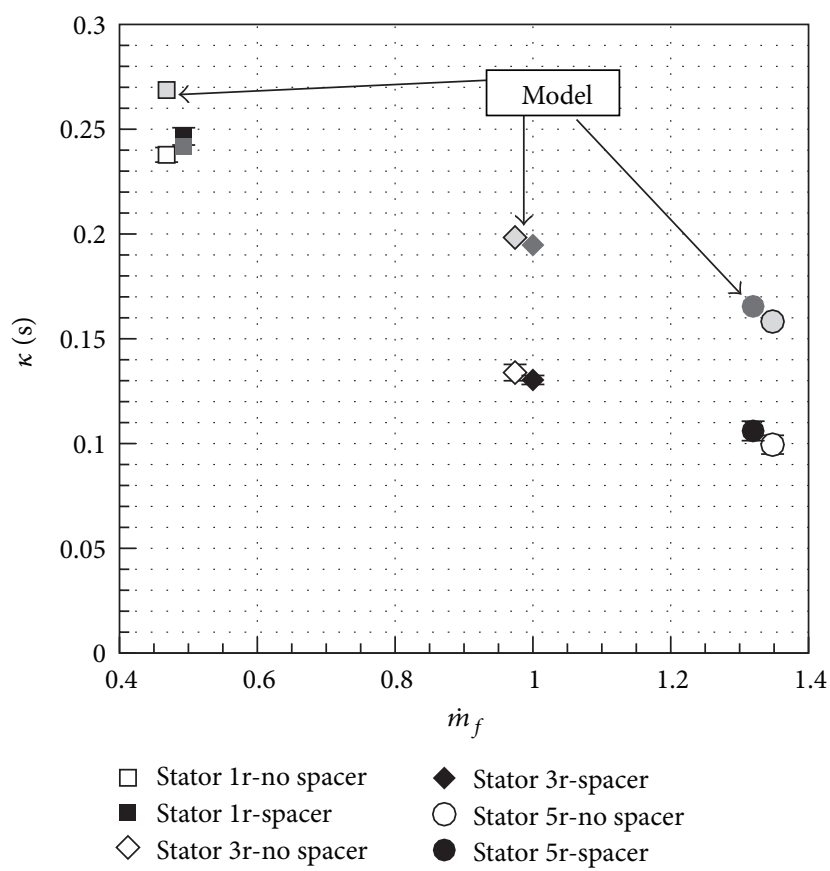

(b) Response time as a function of the mass-flow of the final operating point: comparison of the theoretical prediction (12) and experimental results

FiguRE 5: Final operating point and reactivity of the stage for three opening configurations of the stator.

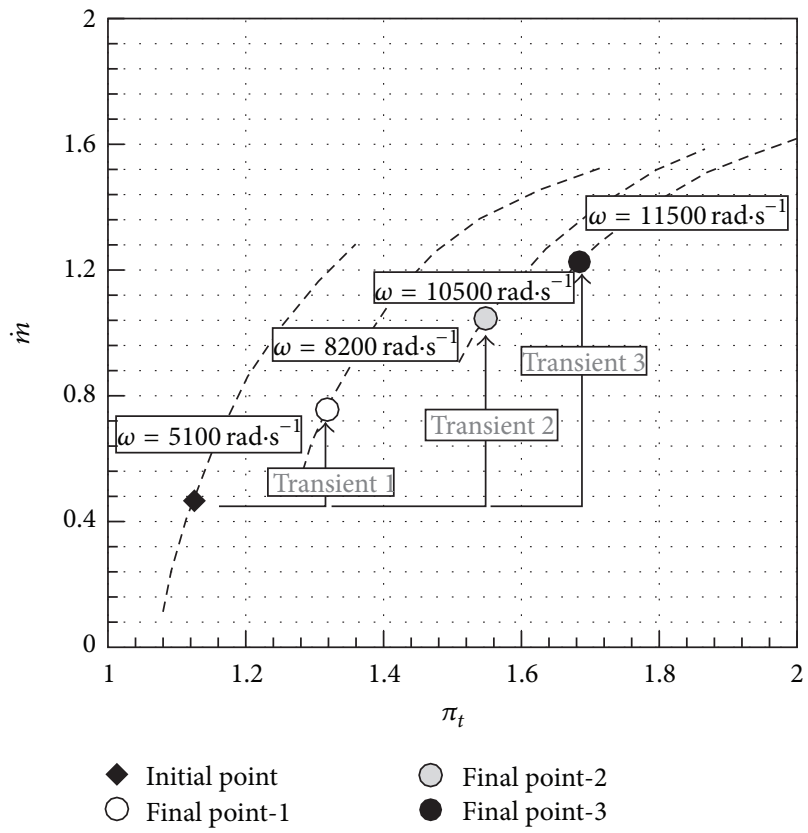

(a) Position of the initial and three final operating points in the turbine map

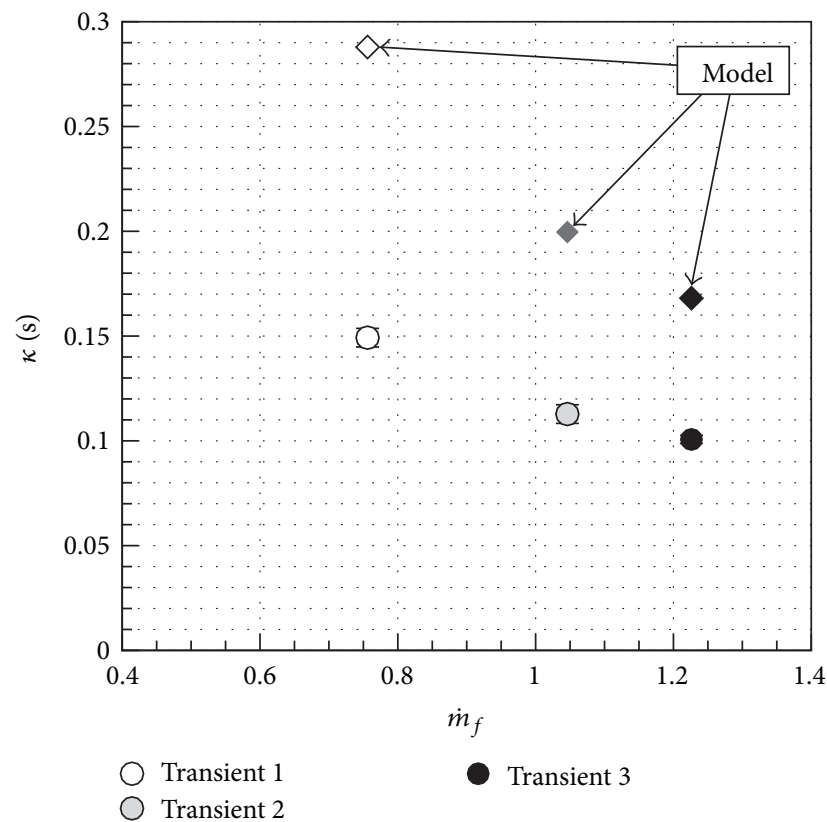

(b) Response time as a function of the final mass-flow

FIgURE 6: Modification of the gap in rotational speed during the transient for the stage $3 h$. 


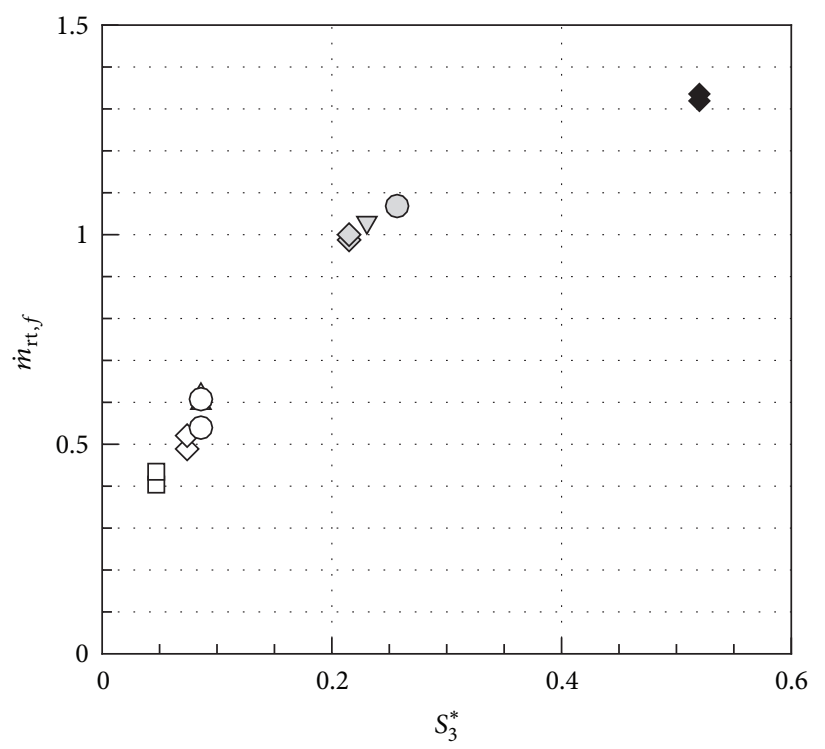

$\begin{array}{ll}\diamond \text { Stator } 1 \mathrm{r} & \triangle \text { Stator } 2 \mathrm{~s} \\ \diamond \text { Stator } 3 \mathrm{r} & \nabla \text { Stator } 4 \mathrm{~s} \\ \diamond \text { Stator } 5 \mathrm{r} & \bigcirc \text { Stator } 1 \mathrm{~h} \\ \square \text { Stator } 1 \mathrm{~s} & \bigcirc \text { Stator } 3 \mathrm{~h}\end{array}$

(a) Corrected mass-flow rate as a function of the reduced section

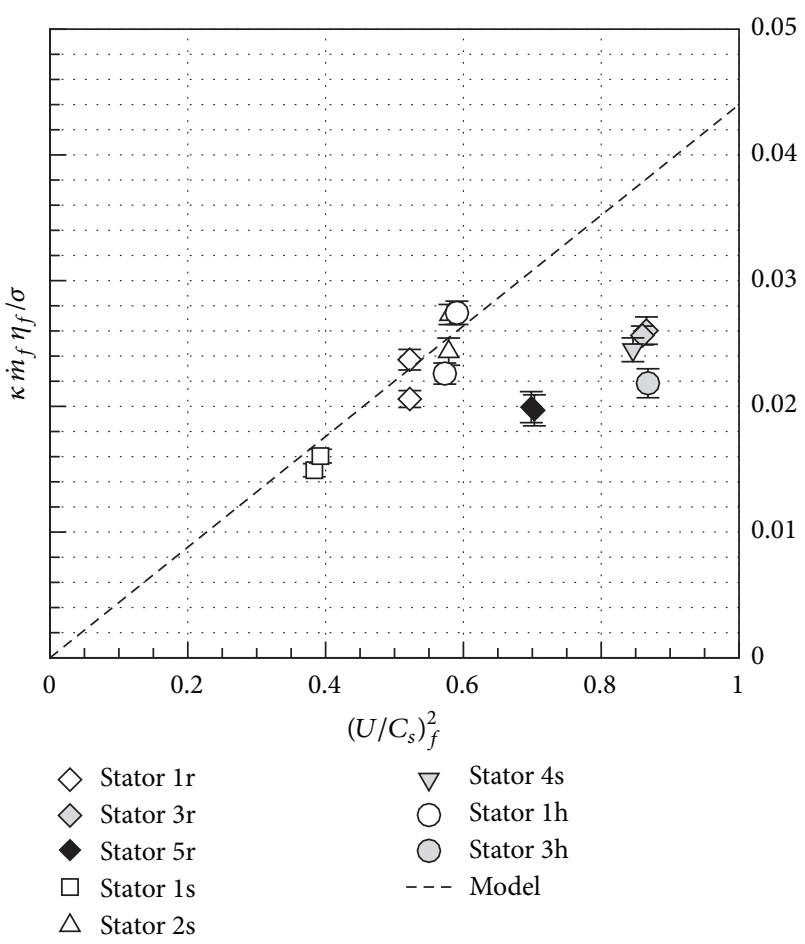

(b) Transient mass as a function of the square of the reduced tip speed

FIGURE 7: Agreement between theoretical prediction and experimental results for all the configurations, for a final operating point of $\pi_{t}=1.6$.

differ geometrically (see [6] for more details). The reduced section actually defines the permeability of the stage, as long as the minimum section of the stage is in the stator. This classification in terms of reduced section is important, because the validity of the theoretical prediction of $\kappa$ is partially related to it. A reversal formulation of (12) is

$$
\frac{\kappa \dot{m}_{f} \eta_{f}}{\sigma}=8 \frac{J}{D_{4}^{2}}\left(\frac{U_{4}}{C_{s}}\right)_{f}^{2}
$$

This specific formulation is convenient because there is a separation between the response time and the aerodynamic performance on the left-hand side (which are results), and the request in terms of final rotational speed with the characteristics of the shaft on the right-hand side (which are imposed by the transient operation). Both terms of the equation have the dimension of a mass. On the left-hand side, this "transient mass" is roughly the mass of fluid crossing the stage during the transient phase and is supposed to evolve linearly with the square of the final reduced tip speed of the rotor. The slope of the line $\left(8 \mathrm{~J} / D_{4}^{2}\right)$ is a characteristic mass of the shaft, which is common to all configurations. This supposed linearity is checked in Figure 7(b) where the "transient mass" is plotted as a function of the square of the reduced tip speed. What was observed in Figure 5(b) is here generalized to every configuration tested. The experimental results and the theoretical predictions are in a good agreement for some configurations, those having a small value of the reduced section. A strong discrepancy appears for the others, when the reduced section of the geometry is increased. The actual response time is then much shorter than the prediction of the quasi-static approach, almost halved. For the theoretical model it means that

(1) either the quasi-static approach is correct, and all the other approximations (such as the decomposition of the transient phase in two subphases, the linearity of the torques, and the estimation of the intermediate torque $\left.\left(\tau_{T, i^{\prime}}\right)\right)$ lead to the deviation of the model;

(2) or those approximations are acceptable but with the quasi-static approach some phenomena in the transient phase are out of reach.

Whatever its origin is, the deviation is inactive for the configurations having a small value of the reduced section and it is very intense for the others. This duality is surprising since the different stages tested are not extremely different from one another in terms of geometry, and the hypotheses of the model are always the same.

Some additional tests have thus been carried out, to incriminate one of the two previous propositions. For the first one, the possible decomposition in two subphases is supported by the literature, and by recent unsteady measurements in the intermediate locations of the stage; it seems reasonable enough. The linearity of the torque during the phase $\left[i^{\prime}-f\right]$ has been checked for the braking torque, by reproducing the expected transient phase through a succession of steady points. In that quasi-static approach, the linearity has been observed. For the turbine, the same approach has not been possible on the complete segment $\left[i^{\prime}-\right.$ $f]$ because of the limitations imposed by the blockage and 


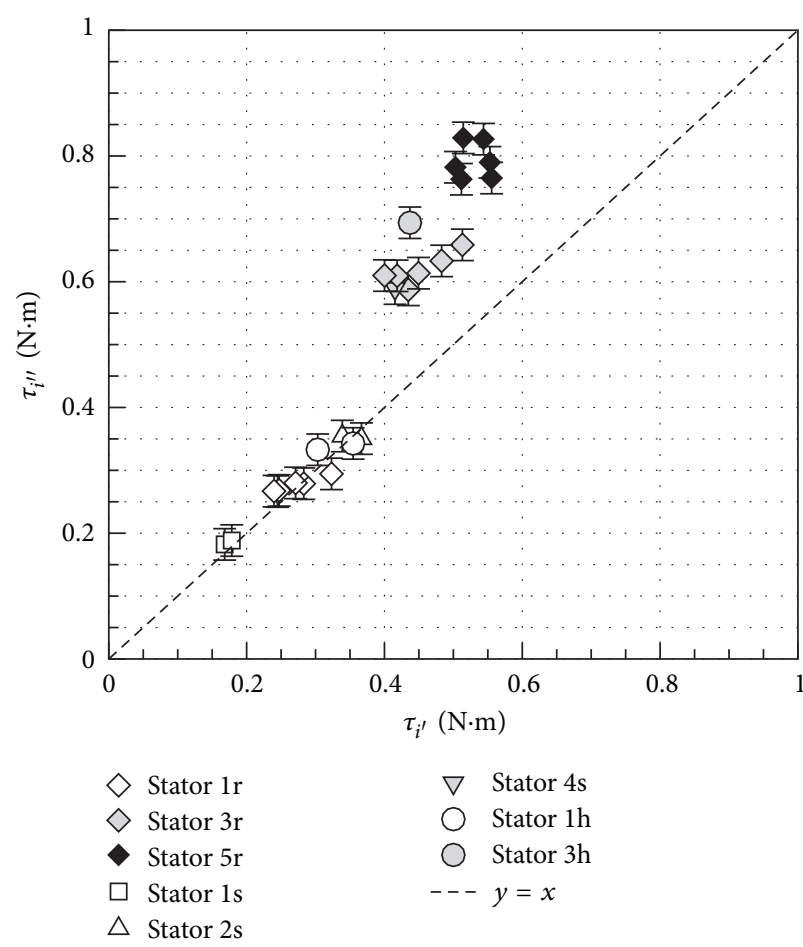

(a) Comparison for the different stator geometries

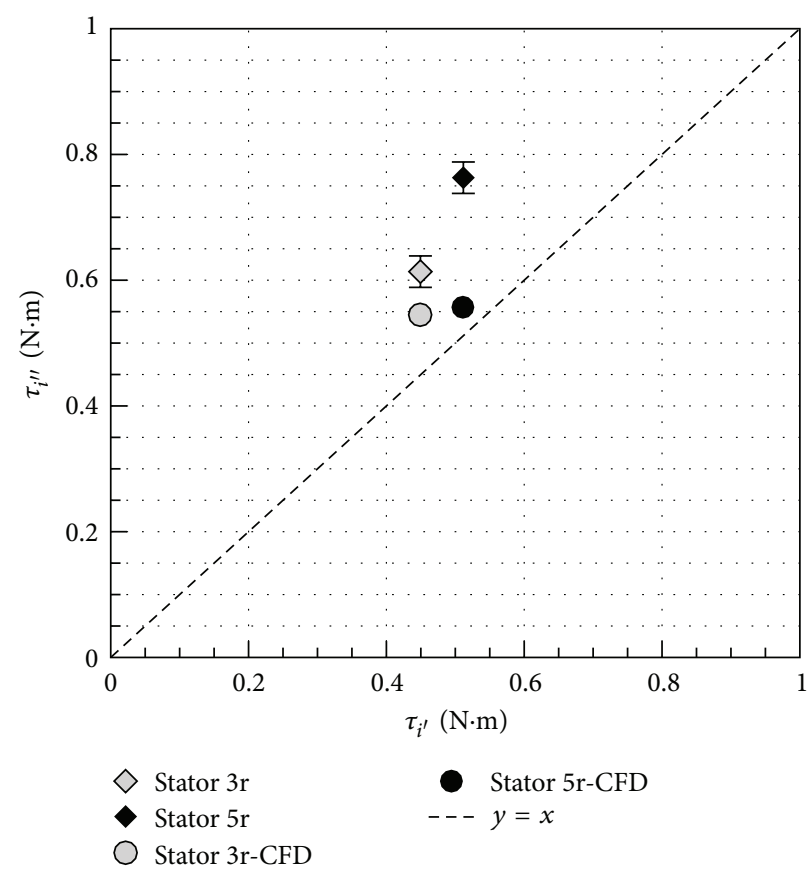

(b) Comparison of the CFD prediction of $\tau_{i^{\prime}}$ with $\tau_{i}^{\prime \prime}$ for two stator geometries

FIGURE 8: Comparison of the initial estimation of the torque at the point $i^{\prime}$ given by (9) with the back-deduction of the torque through the response time $\left(\tau_{i}^{\prime \prime}\right)$.

surge regions of the compressor used as "braking device," but the portion observed was also linear.

Only two possible explanations remain: a strong underestimation of $\tau_{T, i^{\prime}}$ for some configurations and the occurrence of an unsteady phenomenon activating the response time. No measurement of $\tau_{T, i^{\prime}}$ can be reached experimentally (the steady representation of point $i^{\prime}$ corresponds to a high massflow $\left(\dot{m}_{i^{\prime}} \approx \dot{m}_{f}\right)$ and pressure-ratio $\left(\pi_{t, i^{\prime}} \approx \pi_{t, f}\right)$ and a small rotational speed $\left(\omega_{i^{\prime}}=\omega_{i}\right)$; it is far over the possible absorption of energy of our compressor at this rotational speed, for steady conditions). But though (8) it is possible to back-deduce a value of $\tau_{T, i^{\prime}}$ from the experimental results of the response time. This back-deduction quoted $\tau_{T, i}{ }^{\prime \prime}$ is thus the value that the instantaneous torque should take at point $i^{\prime}$ to match the measured response time. It is compared in Figure $8(\mathrm{a})$ to the initial estimation of $\tau_{T, i^{\prime}}$ given by (9) and implemented in the quasi-static model. For the configuration presenting a small reduced-section value (white symbols in Figure 8(a)), as another expression of the good matching between the prediction and the results, we find that $\tau_{T, i}{ }^{\prime \prime} \approx$ $\tau_{T, i^{\prime}}$. For intermediate and high values of the reduced section (grey and black symbols), the torque back deduced from the response time measurement is far more important, up to $30 \%$ more than the approximation. Indeed, the approximation proposed in (9) is quite simplistic and does not have an equal validity for the different stator configurations or the different regions of the map. This validity is checked through the simulation of the operating point $i^{\prime}$ with CFD calculations, since it is out of the possible range of the test bench. Some simulations of the operating points $i$ and $i^{\prime}$ were performed for the stators $3 r$ and $5 r$ (see Section 3 for the details of methodology). The simulation of the point $i$ aims at validating the torque predicted by CFD, compared with the steady results. Then the same methodology and postprocess are applied for the prediction of the torque for $i^{\prime}$. This prediction is hopefully more accurate than (9) for the point $i^{\prime}$ and compared with the results of the quasi-steady approach $\left(\tau_{T, i^{\prime}}\right.$ simulated compared with $\tau_{T, i^{\prime}}$ back-deduced). The results are presented in Figure 8(b) and show that the approximation of (9) is acceptable. More important, even with the proper estimation of $\tau_{T, i^{\prime}}$ the quasi-static prediction still fails to match the measurement, even if it gets closer to it. For the nominal configuration a gap is still present. For the fullopened configuration, the torque at point $i^{\prime}$ is still more than $20 \%$ lower than the value compatible with the measured response time.

Summarizing, the quasi-steady inaccuracy increases as the characteristic time of the transient decreases. Since the short response-time cases are also the ones for which the mass-flow is important, this inaccuracy is observed for the configurations presenting the less restrictive stator geometries (full-opened geometries). The fact that the response time is significantly shorter than the one predicted is noteworthy. Thus the possibility of a phenomenon out of the scope of 
the quasi-steady approach rises but still needs to be verified. The creation of an unsteady "overtorque" due to the passage of the pressure front across the stage during the brutal transient could be considered. Since the pressure front is probably damaged by the very restrictive closed nozzles, it is not active for the small reduced-section configurations. When the front keeps some coherence through the stator, the transient phenomenon appears, and the torque $\tau_{i^{\prime}}$ is reinforced to an instantaneous value $\tau_{i}{ }^{\prime \prime}$. This has not been demonstrated and is still speculative. It has not been observed but it is not surprising since most of the unsteady results found in the literature concern periodic pulsating flows, for nominal geometries of the stator (for those configurations the transient phenomenon is not strongly active).

\section{Conclusion}

Some measurements of the transient response of a variable geometry turbine have been conducted and compared to some theoretical predictions. The matching is good for some opening configurations of the stator, less for others. More specifically when the reduced section of the stator is important, some very strong reactivity has been observed. The different conclusions can be summarized as follows.

(1) It is possible to change the transient response of a shaft without changing its polar moment of inertia. A substantial gain of efficiency will produce a gain of reactivity.

(2) The main factor is the mass flow rate. To increase the reactivity of a given shaft through a transient operation, the most efficient way is to increase the mass flow level. The geometry of the stator should be adapted to the increase of mass-flow rate.

(3) For configurations presenting an important value of the reduced section, the actual response time is significantly smaller than the quasi-steady prediction.

(4) An unsteady creation of torque is suspected, if not demonstrated. The origin of this "overtorque" is conjecturally related to the passage of the pressure front.

(5) If the geometry of the stator is too restrictive, the "overtorque" is ineffective and the quasi-steady approach is accurate enough to predict correctly the response time.

This process needs to be verified in detail. But should it be demonstrated, this could explain some of the unsteady measurements of efficiency presented in the literature which are above 1 , even if it is also agreed that the inappropriate definition of the efficiency in an unsteady context is also part of such amazing results.

\section{Nomenclature}

\section{Quantities}

$C_{p}:$ Specific heat at constant pressure $(\mathrm{J} /(\mathrm{kg} \cdot \mathrm{K}))$

$C_{s}$ : Isentropic speed $(\mathrm{m} / \mathrm{s})$
$D_{4}$ : Rotor inlet diameter $(\mathrm{m})$

$H$ : Stator-blade opening height $(\mathrm{m})$

$J: \quad$ Mass-moment of inertia $\left(\mathrm{kg} \cdot \mathrm{m}^{2}\right)$

$S_{3}$ : Stator throat section

$S_{3}^{*}$ : Stator reduced section

T: Temperature $(\mathrm{K})$

$t$ : Time (s)

$U_{4}$ : Rotor inlet tip speed $(\mathrm{m} / \mathrm{s})$

$\dot{W}$ : Power (W)

$\dot{m}$ : Mass-flow rate $(\mathrm{kg} / \mathrm{s})$

$\alpha$ : Stator-blade opening angle

$\omega$ : Rotational speed ( $\mathrm{rad} / \mathrm{s})$

$\eta$ : Efficiency

$\gamma: \quad$ Specific heat ratio

$\kappa$ : Response time (s)

$\pi_{T}$ : Turbine pressure ratio

$\tau: \quad$ Torque $(\mathrm{N} \cdot \mathrm{m})$

$\sigma$ : Transient parameter.

\section{Subscripts}

$B$ : Relative to the braking device

$f$ : Final point of the transient

$i$ : Initial point of the transient

il: Intermediate point of the transient

$r t$ : Reduced quantity

$t$ : Total state

$T$ : Relative to the turbine.

\section{References}

[1] H. Moustapha, M. Zelesky, N. C. Baines, and D. Japiske, Axial and Radial Turbines, Concepts ETI, 2003.

[2] B. Lakshminarayana, Fluid Dynamics and Heat Transfer of Turbomachinery, John Wiley and Sons, New York, NY, USA, 1996.

[3] G. Cox, A. Roberts, and M. Casey, "The development of a deviation model for radial and mixed-flow turbines for use in throughflow calculations," in Proceedings of the ASME Turbo Expo Conference, vol. 7, pp. 1361-1374, Orlando, Fla, USA, June 2009.

[4] R. H. Aungier, Turbine Aerodynamics: Axial-Flow and RadialinFlow Turbine Design and Analysis, ASME press, 2006.

[5] A. Whitfield and N. C. Baines, Design of Radial Turbomachines, Longman Scientific and Technical, New York, NY, USA, 1990.

[6] N. Binder, S. le Guyader, and X. Carbonneau, "Analysis of the variable geometry effect in radial turbines," Journal of Turbomachinery, vol. 134, no. 4, Article ID 041017, 9 pages, 2011.

[7] D. E. Winterbone and R. J. Pearson, "Turbocharger turbine performance under unsteady flow-a review of experimental results and proposed models," In IMechE C554/031, 1998.

[8] N. C. Baines, Turbocharger Turbine Pulse Flow Performance and Modelling-25 Years on, Concepts NREC, 2010.

[9] D. A. Ehrlich, Characterization of unsteady on-engine turbocharger turbine performance [Purdue University Thesis], 1998.

[10] C. D. Rakopoulos and E. G. Giakoumis, "Availability analysis of a turbocharged diesel engine operating under transient load conditions," Energy, vol. 29, no. 8, pp. 1085-1104, 2004. 
[11] G. G. Venson and J. E. M. Barros, "Turbocharger dynamic analysis using first order sytem step response," In GT200959822, 2009.

[12] N. Watson and S. Janota, Turbocharging the Internal Combustion Engine, MacMillan, New York, NY, USA, 1982.

[13] R. Barkhage, Evaluation of a variable Nozzle turbine turbocharger on a diesel engine under steady and transient conditions [Ph.D. thesis], Chalmers University of Technology, GÃuteborg, Sweden, 2002.

[14] C. Brüstle, J. Wagner, K. T. Van, and K. Burk, “Turbocharging techniques for sports car engines," IMechE C405/055, 1990.

[15] Z. Filipi, Y. Wang, and D. Assanis, "Effect of variable geometry turbine (vgt) on diesel engine and vehicle system transient response," SAE Paper 2001-01-1247, 2001.

[16] H. Uchida, "Transient performance prediction for turbocharging systems incorporating variable-geometry turbochargers," R\&D Review of Toyota CRDL, vol. 41, no. 3, 2006.

[17] A. Suresh, D. C. Hofer, and V. E. Tangirala, "Turbine efficiency for unsteady, periodic flows," Journal of Turbomachinery, vol. 134, no. 3, Article ID 034501, 6 pages, 2011.

[18] N. Binder, X. Carbonneau, and P. Chassaing, "Influence of a variable guide vane nozzle on the design parameters of a radial turbine stage," in Proceedings of the 6th European Conference on Turbomachinery, Fluid Dynamics and Thermodynamics, Lille, France, 2005.

[19] P. L. Meitner and A. J. Glassman, "Off-design performance loss model for radial turbine with pivoting variable-area stators," NASA-TP- 1708, 1980. 

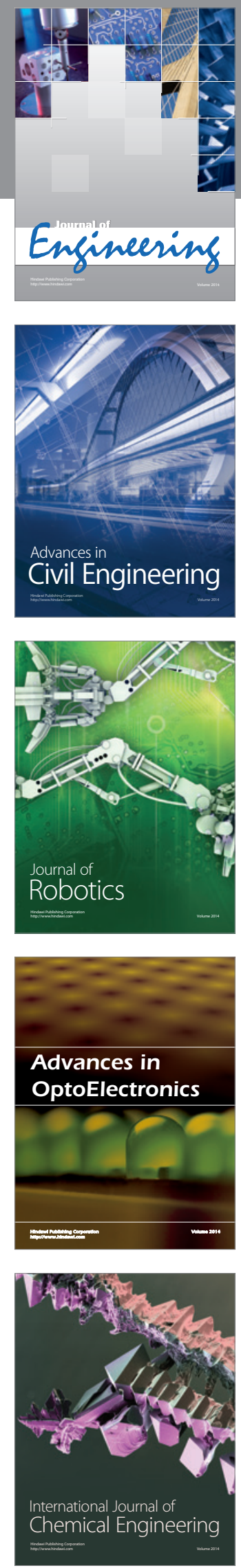

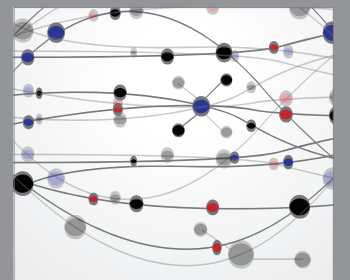

The Scientific World Journal
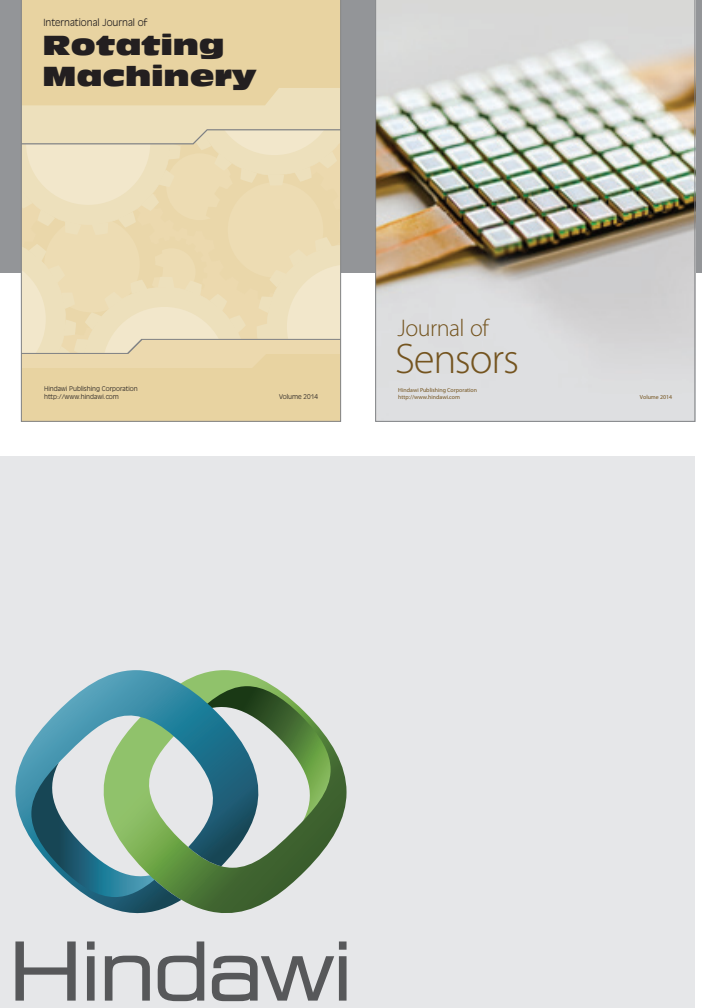

Submit your manuscripts at http://www.hindawi.com
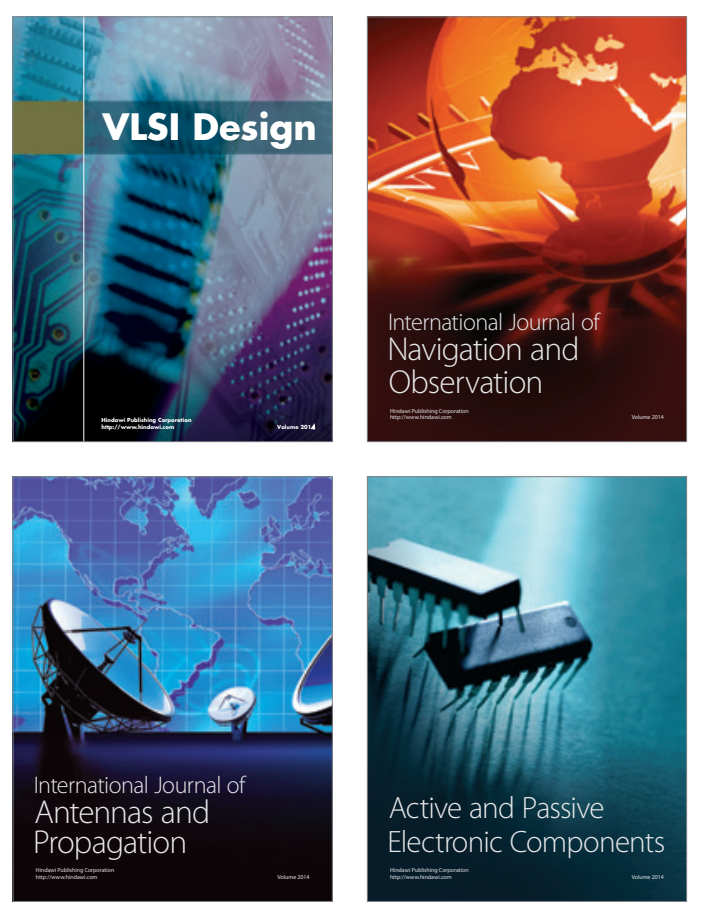
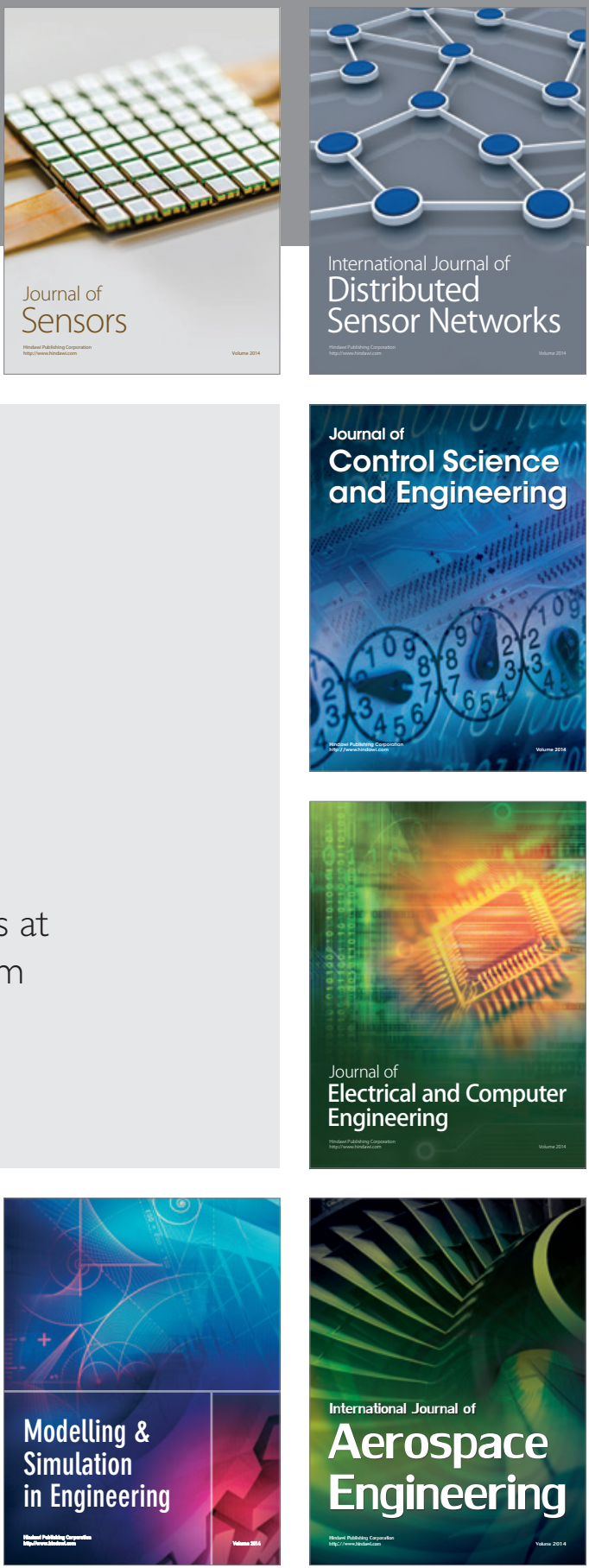

Journal of

Control Science

and Engineering
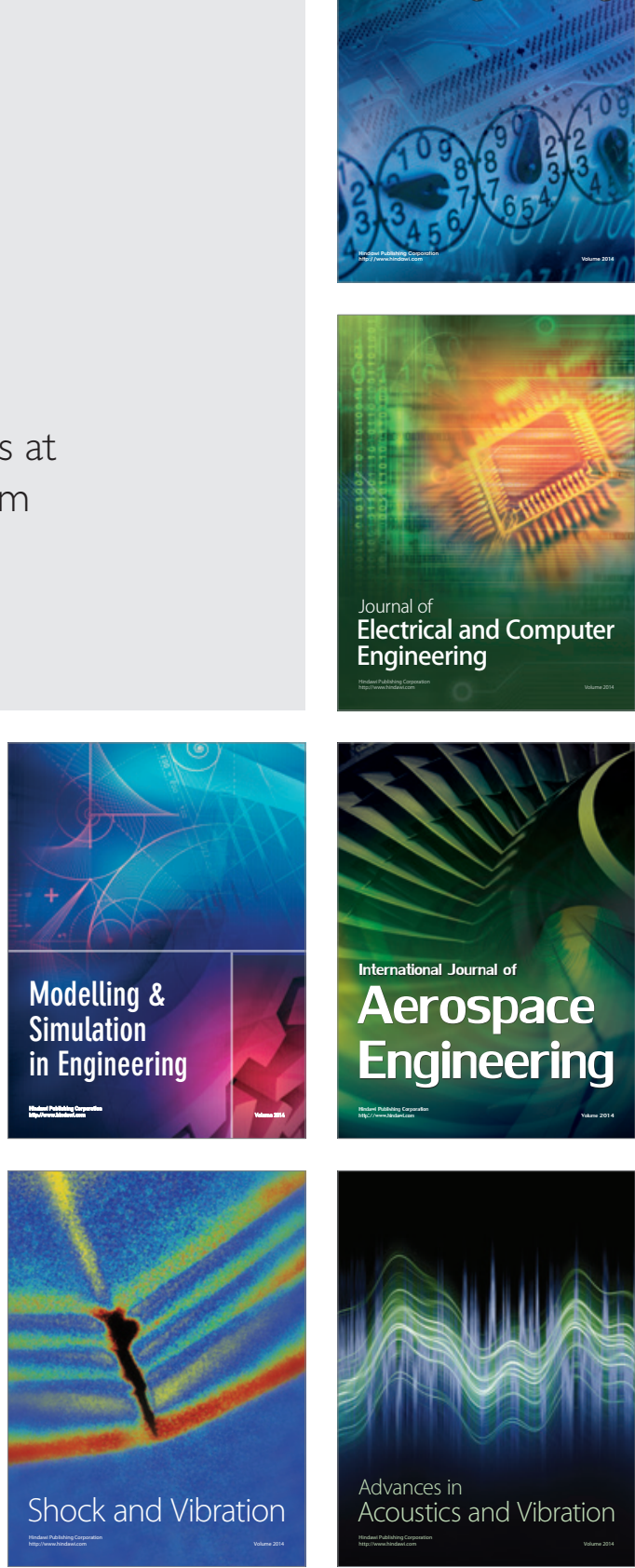